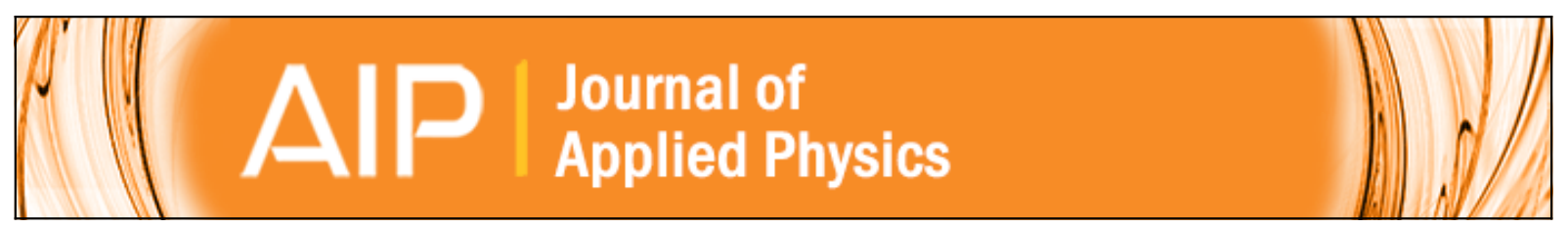

\title{
Morphology and properties of a hybrid organic-inorganic system: Al nanoparticles embedded into CuPc thin film
}

O. V. Molodtsova, I. M. Aristova, S. V. Babenkov, O. V. Vilkov, and V. Yu. Aristov

Citation: Journal of Applied Physics 115, 164310 (2014); doi: 10.1063/1.4874161

View online: http://dx.doi.org/10.1063/1.4874161

View Table of Contents: http://scitation.aip.org/content/aip/journal/jap/115/16?ver=pdfcov

Published by the AIP Publishing

\section{Articles you may be interested in}

One-dimensional array of point-like light sources based on gold nanoparticles and tetracene: Preparation and possible operation mechanisms

Appl. Phys. Lett. 105, 193302 (2014); 10.1063/1.4901748

Investigation of Ag-poly(3,4-ethylenedioxythiophene):polystyrene sulfonate nanocomposite films prepared by a one-step aqueous method

J. Appl. Phys. 109, 124902 (2011); 10.1063/1.3596570

Communication: Unusual dynamics of hybrid nanoparticles and their binary mixtures

J. Chem. Phys. 133, 151105 (2010); 10.1063/1.3495480

Properties of hybrid organic-inorganic systems: Au nanoparticles embedded into an organic CuPc matrix Appl. Phys. Lett. 97, 113103 (2010); 10.1063/1.3488809

Real-time monitoring of the evolving morphology and molecular structure at an organic-inorganic semiconductor interface: SnPc on $\mathrm{GaAs}(001)$

J. Vac. Sci. Technol. B 28, C5F5 (2010); 10.1116/1.3464769

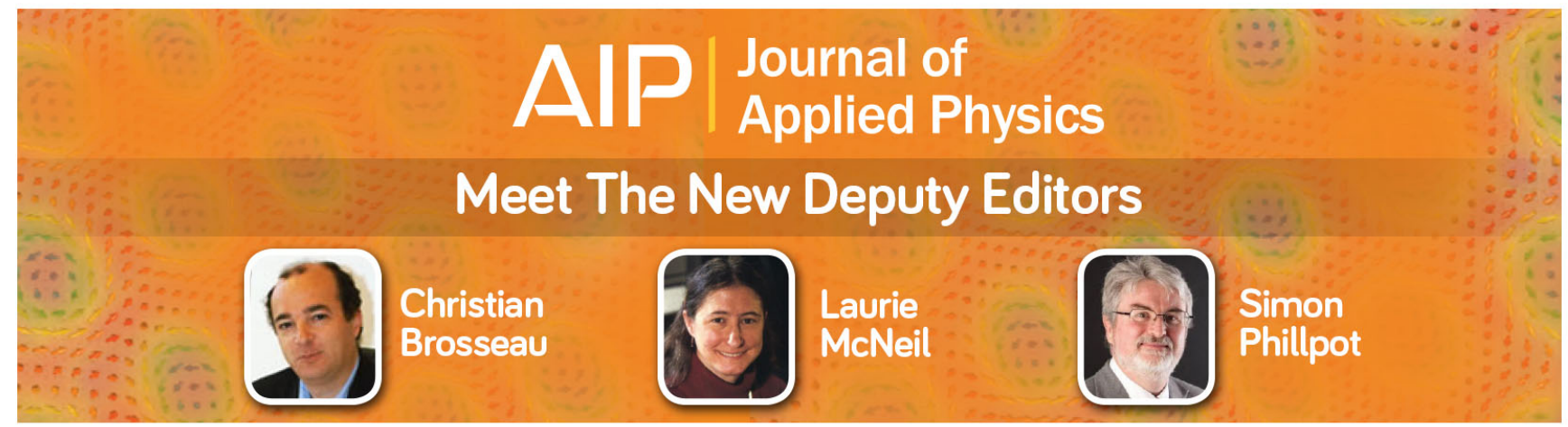




\title{
Morphology and properties of a hybrid organic-inorganic system: Al nanoparticles embedded into CuPc thin film
}

\author{
O. V. Molodtsova, ${ }^{1}$ I. M. Aristova, ${ }^{2}$ S. V. Babenkov, ${ }^{1}$ O. V. Vilkov, ${ }^{3}$ and V. Yu. Aristov ${ }^{1,2,4, a)}$ \\ ${ }^{1}$ Deutsches Elektronen-Synchrotron DESY, Notkestraße 85, 22607 Hamburg, Germany \\ ${ }^{2}$ Institute of Solid State Physics of Russian Academy of Sciences, Chernogolovka 142432, Russia \\ ${ }^{3}$ Helmholtz-Zentrum Berlin (HZB) für Materialien und Energie, Albert-Einstein-Straße 15, 12489 Berlin, \\ Germany \\ ${ }^{4}$ Institut für Theoretische Physik, Universität Hamburg, Jungiusstraße 9, D-20355 Hamburg, Germany
}

(Received 11 March 2014; accepted 18 April 2014; published online 29 April 2014)

\begin{abstract}
The evolution of the morphology and the electronic structure of the hybrid organic-inorganic system composed of aluminum nanoparticles (NPs) distributed in an organic semiconductor matrix - copper phthalocyanine $(\mathrm{CuPc}$ — as a function of nominal aluminum content was studied by transmission electron microscopy and by photoemission spectroscopy methods. The aluminum atoms deposited onto the CuPc surface diffuse into the organic matrix and self-assemble to NPs in a well-defined manner with a narrow diameter distribution, which depends on the amount of aluminum that is evaporated onto the $\mathrm{CuPc}$ film. We find clear evidence of a charge transfer from $\mathrm{Al}$ to $\mathrm{CuPc}$ and we have been able to determine the lattice sites where Al ions sit. The finally at high coverage about $64 \AA$ the formation of metallic aluminum overlayer on CuPc thin film takes place. (C) 2014 AIP Publishing LLC. [http://dx.doi.org/10.1063/1.4874161]
\end{abstract}

\section{INTRODUCTION}

Nowadays, when information technology has become an important part of our lives and the complexity of the mobile gadgets increases the attention is paid for more efficient and faster memory structures. Super miniaturization with extremely small bit cell size from micro-scale to nano-scale and data storage become important issues. There are tremendous worldwide efforts to develop new memory devices for long-term storage of the information. ${ }^{1,2}$ The new kind of memory based on switchable resistive materials is commonly ranked as resistive random-access memory (RRAM). Hybrid systems, mainly consisting of inorganic nanoparticles (NPs) blended into an organic matrix, have been proposed as one type of RRAM. ${ }^{3-6}$

Possible memory architecture is obtained if an organic thin film containing metal nanoparticles is sandwiched between a cross-point array of electrodes-that consist of narrow metal stripes, running in perpendicular directions above and below the film (see Fig. 1).

The resistivity at "cross points" can be switched either a high- or low-conductivity state by applying a voltage, suitable to write or erase. Above a threshold voltage, the device suddenly switches from a high-impedance state to a lowimpedance state and remains in that state even when the power is off. The resistivity in the high- and low conductivity state can differ by $6-8$ orders of magnitude. ${ }^{7,8}$ The highimpedance state can be recovered by applying a voltage in the reverse direction. The corresponding state can then be probed by measuring the current across the cross points at some lower voltages. By defining the two states as " 1 " and " 0, " it is possible to create digital memory devices. These two states can be viewed as the realization of non-volatile

${ }^{a)}$ Electronic mail: aristov@issp.ac.ru electrical memory, thus rendering the structures suitable for data storage.

Albeit, there is a rapid development in this area but the precise memory mechanism is still unclear and many questions left unanswered. ${ }^{5,9,10}$ Properties of the materials formed with participation of nanometer-sized (tens or units nanometers) structural elements, are not identical with the properties of bulk matter, so it is possible to consider such nanostructures as a special state of substance.

The properties of such composite films are strongly linked to the particles nanostructure, i.e., the size, concentration, bulk- and size-distribution of respective nanoparticles. The most fertile model for mechanism that is responsible for the large difference in resistivity between the high and low conductivity states is based on charging of the NPs, which leads to strong modifications of the properties of the organic matrix material (see, e.g., Review 8 and references therein). It is impossible to understand the underlying mechanism without comprehensive information about the specific electron structure and interface interactions between the NPs and the thin organic film matrix. Preliminary studies suggest that

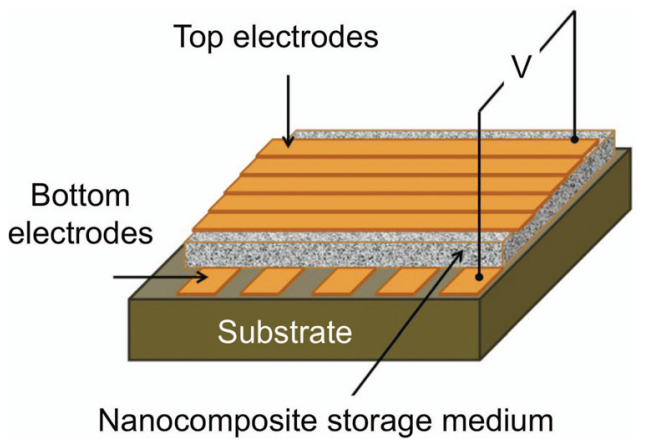

FIG. 1. Cross point memory architecture. 
charge accumulation on nanoparticles can occur provided the nanoparticle energy levels lie within the energy gap of the organic molecules. ${ }^{2}$

The aim of the present work was to investigate the diverse morphologically defined hybrid systems composed of Al NPs distributed in a organic semiconductor copper phthalocyanine matrix, which is formed by molecules with a wide energy gap and delocalized $\pi$-orbitals. The molecular structure of $\mathrm{CuPc}\left(\mathrm{C}_{32} \mathrm{H}_{16} \mathrm{~N}_{8} \mathrm{Cu}\right)$ is schematically shown in Fig. 2. It has a planar structure with $\mathrm{D}_{4 \mathrm{~h}}$ point symmetry. The molecule consists of the central $\mathrm{Cu}$ atom surrounded by four nitrogen atoms (pyrrole, $\mathrm{N}_{1}$ ); four other nitrogen atoms-(bridging aza, $\mathrm{N}_{2}$ ); 32 carbon atoms-the pyrrole $\left(\mathrm{C}_{1}\right)$ and the benzenes $\left(\mathrm{C}_{2}, \mathrm{C}_{3}\right.$, and $\left.\mathrm{C}_{4}\right)$.

\section{EXPERIMENTAL DETAILS}

The organic semiconductor copper phthalocyanine matrix, $\mathrm{CuPc}$ thin film, for hybrid organic-inorganic system was grown on an atomically clean $\mathrm{Au}(001)$ surface. Repeated sputtering and annealing cycles were used for preparing gold surface so that well known $5 \times 20$ surface reconstruction ${ }^{11,12}$ was observed by low energy electron diffraction (LEED). Furthermore, no residual contamination was detected by core-level (CL) photoemission spectroscopy (PES).

Morphology and ordering of the CuPc overlayer were evaluated by means of near-edge x-ray absorption fine structure spectroscopy (NEXAFS) and LEED described in Refs. 13-16. These investigations show, that the CuPc molecules are well ordered with the molecular planes lying parallel to the $\mathrm{Au}(001)$ substrate with a square unit cell of approximately $14 \AA \times 14 \AA$.

A high-purity aluminum was deposited on the outer surface of the organic film in ultra high vacuum (UHV) conditions. The deposition rate was measured by a quartz thickness monitor placed in the position closed to the sample at the same distance from the metal evaporator. Due to surface and bulk diffusion of deposited $\mathrm{Al}$ atoms, the embedded aluminum nanoparticles were self-assembled forming a three-dimensional Al NPs distribution in the bulk of the organic semiconductor.

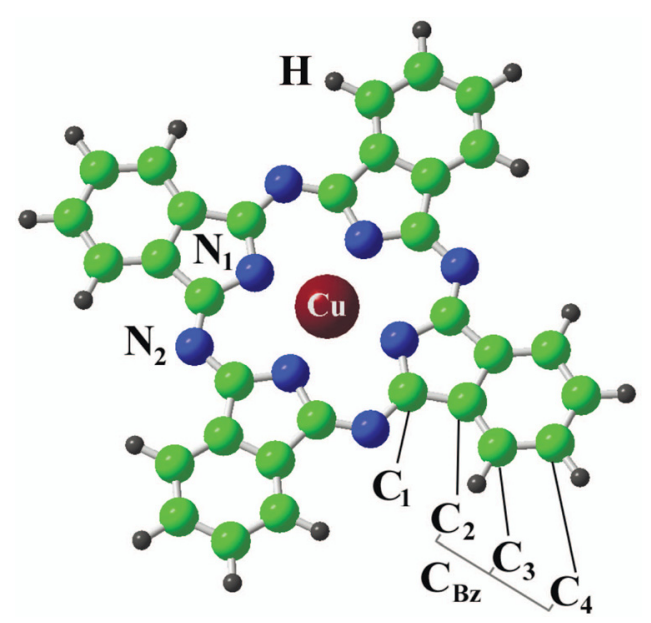

FIG. 2. Schematic representation of the molecular structure of the CuPc molecule, where the central $\mathrm{Cu}$ atom is coordinated to four nitrogen atoms.
The electronic structure of the hybrid organic-inorganic systems, the energy level alignment at interfaces formed between Al NPs and the organic semiconductor $\mathrm{CuPc}$, as well as the chemical interaction at this interface were studied by PES using synchrotron radiation.

The work function $(\Phi)$ changes induced by aluminum deposition on $\mathrm{CuPc}$ thin films were determined from PES data using the cut-off procedure. All PES measurements were performed at the Russian-German high-energy resolution dipole beam line of the Berliner Electronen Speicherring für Synchrotronstrahlung (BESSY, Berlin).

The size, concentration, size-distribution, and shape of the resulting nanoparticles were studied using transmission electron microscopy (TEM) JEM $100 \mathrm{CX}$ operated at $100 \mathrm{kV}$. Thus, the microstructure and evolution of the morphology of the nano-composite films as a function of nominal aluminum coverage were studied similar to described in Ref. 17. The hybrid organic ( $\mathrm{CuPc})$-inorganic (Al) systems for TEM investigations were prepared under UHV conditions on cleaved $\mathrm{NaCl}$ single crystals as substrate using the same UHV chamber, evaporators and the same parameters (temperature, rate of $\mathrm{CuPc}$ deposition, organic film thickness, rate of aluminum deposition, nominal aluminum coverage, etc.) as for the systems grown on $\mathrm{Au}(001)$ surface. In order to bind the nano-composite thin films, the samples were coated by an ultra thin overlayer of amorphous carbon. Film separation from a substrate always occurs easily by dissolution salt in water. Preliminary the film was cut by square pieces with the side of about $2-3 \mathrm{~mm}$. Then, samples are transferred on 250-mesh (250 lines/in.) copper grids for TEM investigations.

\section{RESULTS AND DISCUSSION}

Fig. 3 presents bright-field TEM images of nanocomposite thin films microstructure. It composed of aluminum particles (the black dots in the picture) embedded in an organic semiconductor matrix $\mathrm{CuPc}$ for nominal $\mathrm{Al}$ deposition of $10 \AA$ and $35 \AA$ (see Figs. 3(a) and 3(b), correspondingly).

The right inset in Fig. 3(b) shows an electron diffraction pattern for selected area of the sample with corresponding aluminum deposition and proves that the diffraction originates from aluminum NPs. The left inset in 3(b) shows zoom of an aluminum grain. Fig. 3 demonstrates that the size of the Al NPs strongly depends on aluminum coverage. For nominal $\mathrm{Al}$ deposition of $10 \AA 3$ (a) aluminum nanoparticles form some particle distribution with size about 5-6 nm. Metal nanoparticles are spherical and they are sufficiently described by their mean particle diameter $D=2 R$. With further deposition, one observes strong growth of individual grains. Large particles have a distinct faceting. The size distributions of aluminum nanoparticles corresponding to the electron micrographs of Fig. 3 are shown by histograms in Figs. 3(c) and 3(d). The statistical analysis more than 500 nanoparticles for each image was done.

It was found for nominal coverage of $10 \AA$ aluminum that the averaged diameter of the particles amounts to $\langle\mathrm{d}\rangle=5.7 \mathrm{~nm}$ with a standard deviation of $1.8 \mathrm{~nm}$ and the maximum particle's diameter $14.3 \mathrm{~nm}$. For nominal coverage 

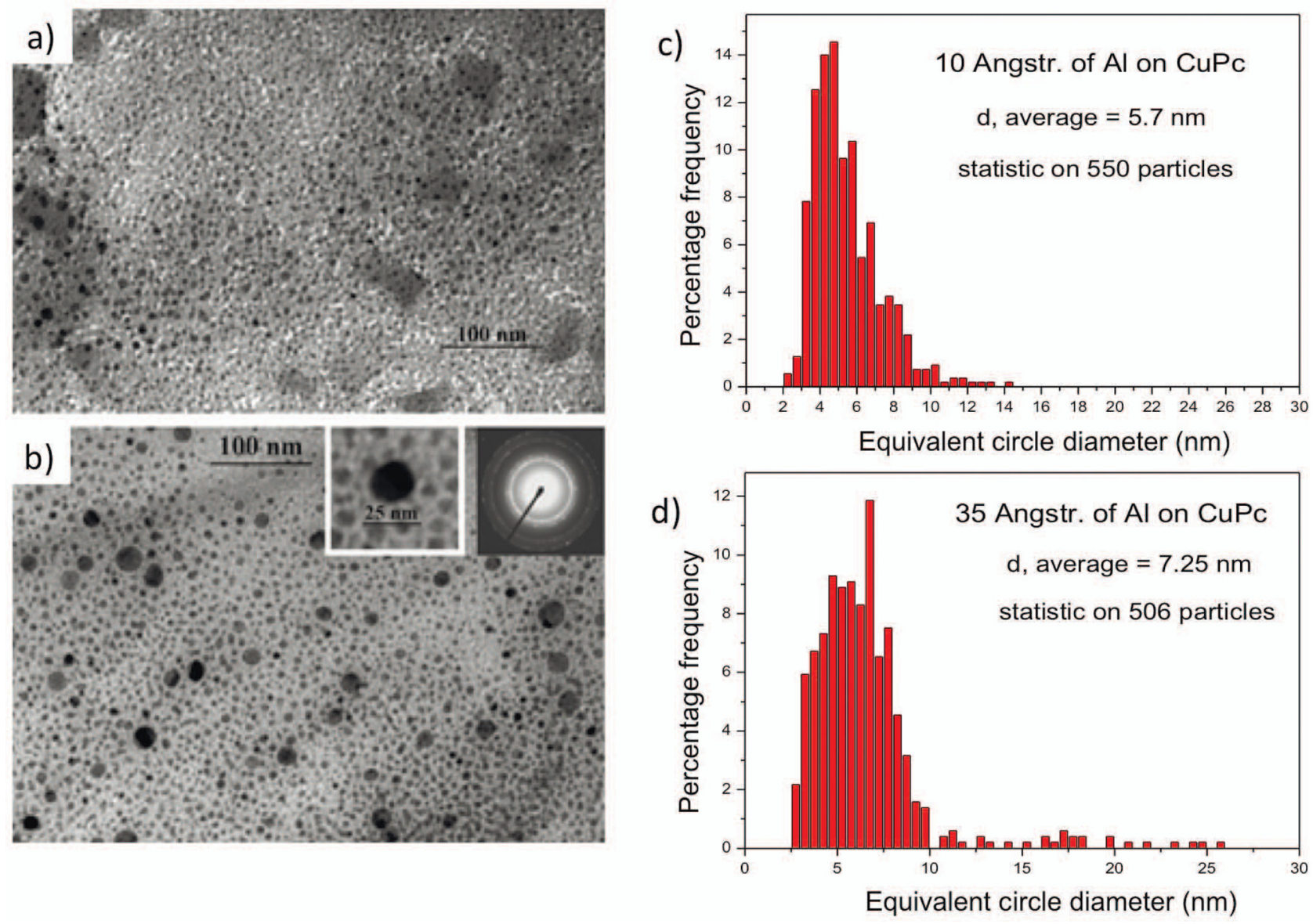

FIG. 3. Microstructure of nanocomposite thin films composed of aluminum particles embedded in CuPc matrix (TEM). Nominal Al deposition is $10 \AA$ (a) and $35 \AA$ (b). Inset in (b): right—electron diffraction patterns for corresponding aluminum deposition, left—zoom of an aluminum grain. (c) and (d): Histograms showing size distribution of aluminum nanoparticles from electron micrographs of (a) and (b).

of $35 \AA$ aluminum, the averaged diameter of the particles amounts to $\langle\mathrm{d}\rangle=7.25 \mathrm{~nm}$ with a standard deviation of $3.3 \mathrm{~nm}$ and the maximum particle's diameter about $26 \mathrm{~nm}$. A large standard deviation indicates that the data points are far from the mean. To some extend this result parallels that for Au deposition on diindenoperylene (DIP) thin films reported in Refs. 18 and 19. It is necessary to notice that if to consider only the particles which size is in the same range, as for a covering $10 \AA$, i.e., which this case standard deviation reduce progressively and it indicates that the data points size does not exceed $14.5 \mathrm{~nm}$ then average diameter of particles makes $6.0 \mathrm{~nm}$ at standard deviation $1.9 \mathrm{~nm}$. $\mathrm{Al}$ atoms are clustered more closely around the mean and in this region the diameter distribution of the aluminum NPs in the CuPc film for nominal coverage of $35 \AA$ aluminum is sufficiently narrow.

The average size of particles in the range of $15-26 \mathrm{~nm}$ makes $19.4 \mathrm{~nm}$ at standard deviation $3.2 \mathrm{~nm}$. Thus, when nominal coverage of aluminum increases from $10 \AA$ to $35 \AA$, the average size of overwhelming majority of aluminum nanoparticles has grown slightly more, than on $5 \%$. On the other hand, the average size about $4 \%$ of particles has increased in 3-4 times. The analysis of size distribution of aluminum nanoparticles allows to assume the following: due to increase of nominal coverage of aluminum some nearby nanoparticles (because of increase their sizes) come to contact and coalesce, forming larger particles.
Coalescence of two or more small particles in one large particle leads to reduction of particles surface area and therefore to reduction of particles surface energy. Thus, we observe the coalescence process of aluminum nanoparticles embedded in the $\mathrm{CuPc}$ film surface. It leads to increase of average diameter of the particles and thereby has an influence on size distribution of aluminum nanoparticles.

The lowermost diagrams in Figs. 4(b)-4(d) were recorded from the clean $\mathrm{CuPc}$ film grown on the $\mathrm{Au}(001)$ substrate. They evidence that the organic film spectra are characteristic of pristine $\mathrm{CuPc}^{20}$

The decomposition of the lowest spectrum in Fig. 4(b) approves former deductions ${ }^{21-23}$ that the $\mathrm{C} 1 \mathrm{~s}$ core-level of pristine $\mathrm{CuPc}$ consists essentially of two components: B, which corresponds to the aromatic carbons of the benzene rings of $\mathrm{CuPc}$, and $\mathrm{P}$, shifted by $1.4 \mathrm{eV}$ toward higher binding energy $(\mathrm{BE})$, which is originated from the pyrrole carbon linked to the more electronegative nitrogen $\mathrm{N}_{1}$ (four nitrogen atoms linked to the central metallic atom) and $\mathrm{N}_{2}$ (four pyrrole nitrogen atoms). The shoulder $S_{B}$ and the feature $S_{P}$ are the satellites of the $\mathrm{B}$ and $\mathrm{P}$ components, respectively. ${ }^{21-27}$ The ratio of the $\mathrm{B}$ to $\mathrm{P}$ components, taking into account the satellites, is very close to theoretically expected value of 3 . The main N 1 s peak of the lowest spectrum in Fig. 4(d) was fitted by only one component thus confirming previous conclusions $^{20-23}$ that, in the case of $\mathrm{CuPc}$, both nitrogen 

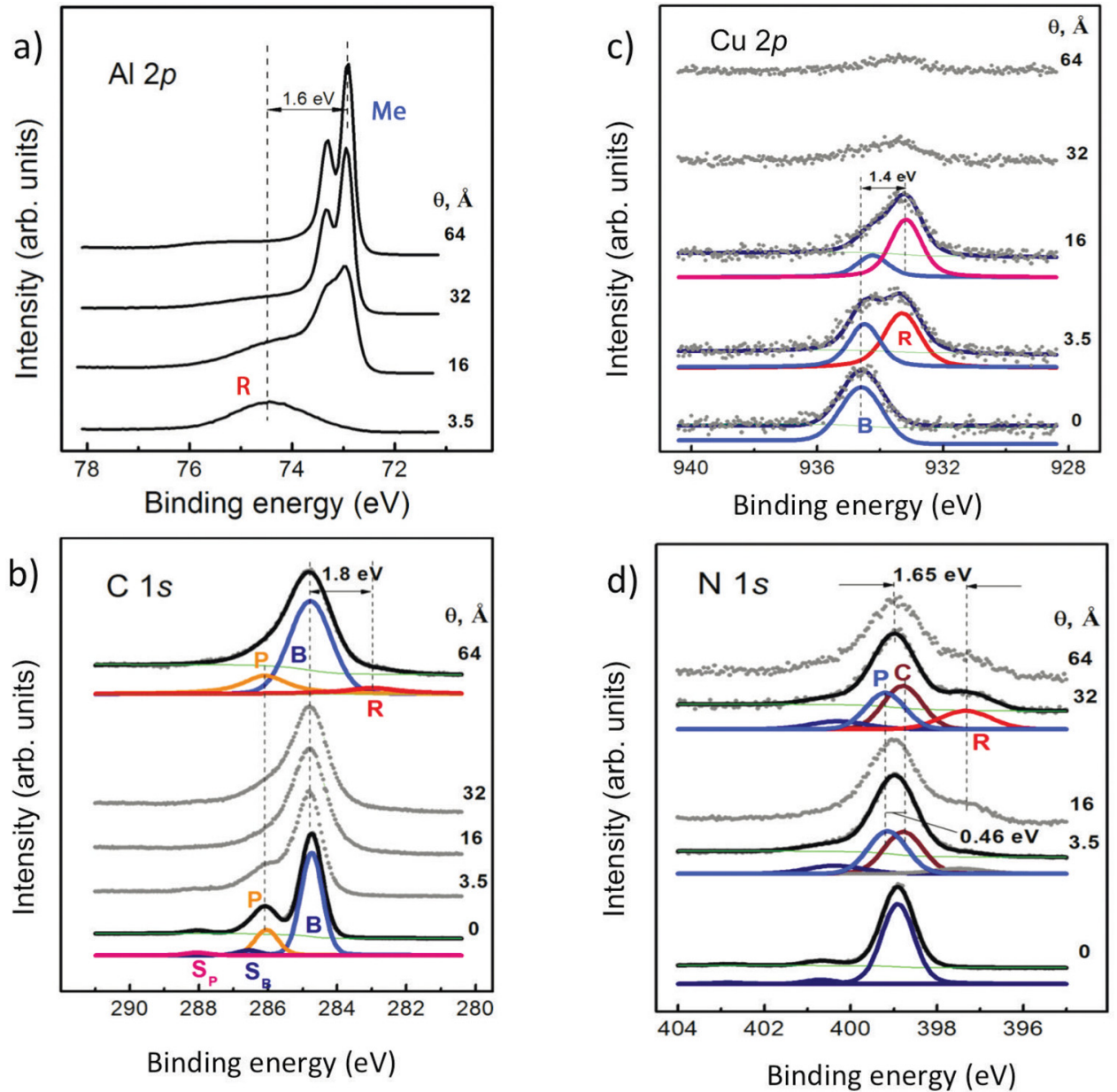

FIG. 4. CL normalized photoemission spectra as a function of $\mathrm{Al}$ deposition on $\mathrm{CuPc}$ thin films at room temperature (RT): $\mathrm{Al} 2 p$ (a), $\mathrm{C} 1 s$ (b), $\mathrm{Cu} 2 p$ (c), and $\mathrm{N} 1 s$ (d). The nominal coverage of the Al deposition is indicated. The behavior of $\mathrm{Cu} 2 p \mathrm{CL}$ is similar to what has been observed for the case of Li, Fe, and $\mathrm{Co}$ deposition onto $\mathrm{CuPc}$ (see below in text).

sites, $\mathrm{N}_{1}$ and $\mathrm{N}_{2}$, are characterized by almost the same binding energy.

The behaviour of the $\mathrm{Al} 2 p$ core level data upon aluminum deposition onto the $\mathrm{CuPc}$ film is presented in Fig. 4(a). At low aluminum coverage, the $\mathrm{Al} 2 p \mathrm{CL}$ shows a strong reactive component $(\mathrm{R})$, which appears in the spectrum shifted by about $1.6 \mathrm{eV}$ to higher $\mathrm{BE}$ with respect to the metallic aluminum doublet. Apparently, this $\mathrm{R}$ component originates from aluminum atoms, which react with CuPc. As a result of this reaction, aluminum atoms transfer charge to $\mathrm{CuPc}$ molecules and become positively charged.

With further deposition, the spectra show appearance of the metallic $\mathrm{Al} 2 p$ component (Me), originated from metallic clusters, which grow very quickly while the relative intensity of the reacted component rapidly decreases which demonstrates the spectrum taken at $16 \AA$ and almost vanishes (spectrum at $32 \AA$ ). Therefore, we have observed two stages of the process of interface formation at $\mathrm{Al}$ deposition onto $\mathrm{CuPc}$ substrate. First stage corresponds to the strong diffusion $\mathrm{Me}$ atoms into organic matrix till a saturation and strong chemical reaction between $\mathrm{Al}$ and $\mathrm{CuPc}$. This stage also characterized by the absence of a detectable density of states near Fermi level $\left(\mathrm{E}_{\mathrm{F}}\right)$ in valence band spectra (not shown). On the second stage, $\mathrm{Al}$ atoms diffuse into organic $\mathrm{CuPc}$ film probably without chemical interaction and form there metallic clusters which is evident from Figs. 4(a) and 4(b). The cluster formation on second stage is confirmed and by formation of significant density of states at $\mathrm{E}_{\mathrm{F}}$ (not shown). Here, we should remark, that similar $\mathrm{Al}$ behavior during deposition onto 3,4,9,10-Perylenetetracarboxylic dianhydride matrix was reported in Ref. 28.

The work function changes as a function of $\mathrm{Al}$ deposition onto organic molecular thin film (CuPC) are presented in Fig. 5. Four special points of curve in this figure- $-\Phi_{\mathrm{Au}}$,

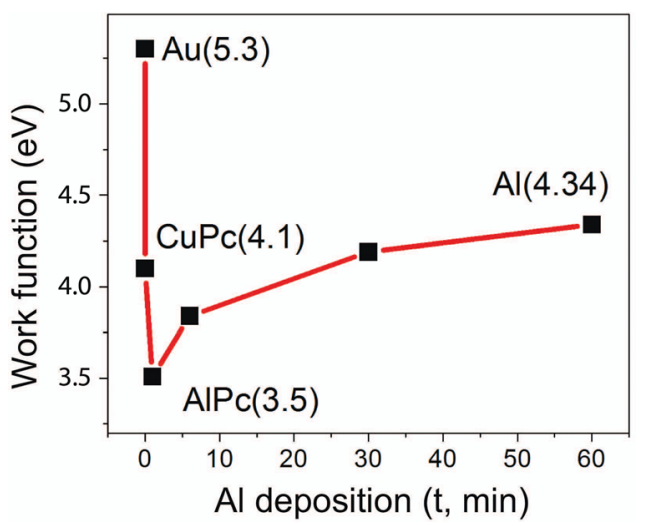

FIG. 5. Work function changes induced by $\mathrm{Al}$ deposition on $\mathrm{CuPc}$ thin films at RT, determined from PES data using the cut-off procedure. 
$\Phi_{\mathrm{CuPc}}, \Phi_{\mathrm{AlPc}}$, and $\Phi_{\mathrm{Al}}$ are typical characteristic work functions of the corresponding materials. ${ }^{29,30}$

From the fact that the work function curve between pristine $\mathrm{CuPc}$ and thick aluminum nominal coverage has a minimum one can suppose that, at low deposition of $\mathrm{Al}$ on the $\mathrm{CuPc}$ surface, the formation of some intermediate phase with work function $\Phi=3.5 \mathrm{eV}$ takes place, which is very close to the work function value $\Phi=3.8 \mathrm{eV}$, measured for AlPc thin films. ${ }^{29,30}$ This assumption confirmed by the $\mathrm{Cu} 2 p$ core level evolution.

To get deeper inside of the possible chemistry at the $\mathrm{Al}$ NPs-CuPc interface, we will now discuss the evolution of the $\mathrm{Cu} 2 p$ core level spectrum upon $\mathrm{Al}$ deposition. Fig. 4(c) shows striking changes in $\mathrm{Cu} 2 p$ spectrum after the first/second steps of aluminum deposition. At very low coverage, a rather strong satellite shoulder of the $\mathrm{Cu} 2 p$ core level appears in the spectrum, shifted by about $1.4 \mathrm{eV}$ to lower BE. Moreover, the relative intensity of this component already at coverage $3.5 \AA$ higher if compare to the intensity of the principal $\mathrm{Cu} 2 p$ peak and therefore one can conclude that the corresponding reaction takes place rather just before the metal clusters formation. Such behavior is evocative of the PES data of $\mathrm{Cu}(\mathrm{I})$ compounds such as $\mathrm{Cu}_{2} \mathrm{O} .{ }^{31}$ Apparently, at this stage of $\mathrm{Al}$ deposition onto $\mathrm{CuPc}$, copper atoms at the subsurface region are reduced from $\mathrm{Cu}(\mathrm{II})$ to $\mathrm{Cu}(\mathrm{I})$, while $\mathrm{Al}$ atoms are oxidized as indicated in Fig. 4(a). The data in Fig. 4(c) would be compatible with a conception where deposited $\mathrm{Al}$ atoms replace $\mathrm{Cu}$ in the center of the phthalocyanine molecules, whereas the released $\mathrm{Cu}$ remains at the aluminum cluster organic matrix interface or segregates near interface as metallic copper clusters. Moreover, $\mathrm{Cu}$ atoms can diffuse to $\mathrm{Al}$ clusters and form the clusters made of $\mathrm{Cu}-\mathrm{Al}$ alloy. The occurrence of such copper atom behavior would parallel the effects of $\mathrm{Li}, \mathrm{Fe}$, and Co deposition onto $\mathrm{CuPc} .^{32-34}$

However, the C $1 s$ and N $1 s$ core level data shown in Figs. 4(b) and 4(d) proof that this conception is too simple and that the phthalocyanine molecule entirely is affected by the interface reactions. Indeed in Ref. 35, it has been suggested that at aluminum deposition onto $\mathrm{F}_{16} \mathrm{CuPc}$ the Al-Pc complexes form, resulting in core level spectra similar to what is evident in Figs. 4(a) and 4(d). Moreover, similar to Ref. 35, one can suppose that reduction of $\mathrm{Cu}$ (II) by $\mathrm{Al}$ could result in formation of the anionic complex $(\mathrm{Cu}(\mathrm{I}) \mathrm{Pc})^{-}$, which could coordinate to the $\mathrm{Al}^{+3}$ ions thus formed through one of the phthalocyanine ring nitrogen lone electron pairs. The overall stoichiometry of the redox reaction product would then be $(\mathrm{CuPc})_{3} \mathrm{Al}$. Indeed, one should consider the possible change of the surface shapes before and after $\mathrm{Al}$ deposition, however, the methods we used in the present investigation do not give us the possibilities to detect such changes.

Now, we think over the deportment of the $\mathrm{C} 1 s$ and $\mathrm{N}$ $1 s$ core level data upon $\mathrm{Al}$ deposition. At the metal deposition, the pyrrole (P) feature of C $1 s$ spectra (Fig. 4(b)) becomes less pronounced, but still persists till aluminum coverage about $3.5 \AA$. These shape changes are accompanied by some enhancement of the background in the same binding energy range. Moreover, the formation of noticeable asymmetric shape of the background was observed with $\mathrm{Al}$ deposition, which could indicate that the outgoing electrons undergo remarkable scattering and thus we can conclude, that apparently formation of large metallic clusters and finally at high coverage a continuous homogeneous metallic overlayer takes place. Here, we should call attention to the fact that the absolute $\mathrm{C} 1 s$ photoemission intensity is also reduced upon metal deposition (see Fig. 4(b)).

At coverages $3.5-15 \AA$, the pyrrole (P) feature of $\mathrm{C} 1 s$ spectrum shows strong degradation and finally can be visible only after C $1 s$ core level decomposition as a component having large width, while the shape of benzene (B) component stays almost unaffected. However, some width enhancement of B component was observed as well.

The conclusions above are supported by the evolution of the N $1 s$ core level (Fig. 4(d)). Even at small coverage of about $3.5 \AA$, spectra show an increase of peak width and the appearance of an additional reactive $\mathrm{N} 1 s$ component (R) at lower BE (about $1.65 \mathrm{eV}$ ). This confirms the strong chemical interaction in low aluminum coverage with the likely formation of iron nitride compounds (e.g., FeN).

To obtain a deeper insight into the chemical interaction between iron and $\mathrm{CuPc}$, we have performed a decomposition of the $\mathrm{N} 1 s$ peak. The bottom N $1 s$ spectrum corresponding to the pristine $\mathrm{CuPc}$ film was fitted by only one component, which is in good agreement with a similar procedure for a clean $\mathrm{CuPc}$ film performed in Refs. 17 and 20, where it was earlier shown that, in the case of $\mathrm{CuPc}$, both nitrogen sites $\left(\mathrm{N}_{1}\right.$ and $\left.\mathrm{N}_{2}\right)$ are characterized by the same or a very similar $\mathrm{N} 1 s \mathrm{BE}$, and the main $\mathrm{N} 1 s$ peak was fitted by only one component describing simultaneously both degenerate types $\left(\mathrm{N}_{1}\right.$ and $\left.\mathrm{N}_{2}\right)$ of nitrogen atoms. Taking into account the fact that we have the same resolution as in Refs. 17 and 20 and taking the same fitting parameters, which were used for CuPc, ${ }^{17,20}$ we analyzed the main N $1 s$ peak by two components: $\mathrm{C}$ (four nitrogen atoms $\mathrm{N}_{1}$ linked to the central metallic atom) and $\mathrm{P}$ (four pyrrole nitrogen atoms $\mathrm{N}_{2}$ ). By assuming that the central $\mathrm{Cu}$ atoms in the vicinity of the $\mathrm{Al} / \mathrm{CuPc}$ interface are replaced by aluminum atoms, one can explain the observed $\mathrm{N} 1 s$ peak splitting. The energy difference between the peaks was found to be about $0.46 \mathrm{eV}$. Component $\mathrm{C}$ at lower $\mathrm{BE}$ can be assigned to the four nitrogen atoms linked to the central metal atom $\left(\mathrm{N}_{1}\right)$. Probably, unlike in the case of $\mathrm{CuPc}$, the central $\mathrm{Al}$ atom more strongly reacts with these four $\mathrm{N}_{1}$ nitrogen atoms, which results an increased charge transfer. The latter provokes the $\mathrm{C}$ component shift to lower $\mathrm{BE}$ by $0.46 \mathrm{eV}$ relative to the $\mathrm{P}$ component. A similar split of about $0.3 \mathrm{eV}$ for $\mathrm{FePc}$ (Ref. 34) was found from DFT calculations, and one of about $0.48 \mathrm{eV}$ for $\mathrm{CoPc}$ molecules from photoemission measurements. ${ }^{35}$ Finally, the $\mathrm{P}$ peak can be assigned as originating from the four nitrogen atoms linked to the pyrrole carbons. Our discussion above is confirmed by metal-Pc BE calculations. ${ }^{36}$ The calculated $\mathrm{BE}$ was found to be $10.49 \mathrm{eV}$ for $\mathrm{CoPc}$, and $6.96 \mathrm{eV}$ for $\mathrm{CuPc}$. These results confirm the fact that the $\mathrm{BE}$ of the metal-Pc is quite sensitive to the nature of the metal.

The analysis of the N $1 s$ spectrum after deposition of $32 \AA$ iron indicates that at this coverage the main spectrum feature still splits into two components and the left one has rather less intensity than the right. It is important to mark 
that significantly increased $\mathrm{N} 1 s$ reacted component R and peak asymmetry. Here, we should emphases that at further deposition the shape of $\mathrm{N} 1 s$ peak remains basically the same however decreases in intensity, which could be explain as the result of formation of the metallic film over the CuPc layer at such coverage. As the left component $(\mathrm{P})$ of the $\mathrm{N}$ $1 s$ core level was attributed to the nitrogen atoms which are linked to pyrrole carbon atoms ${ }^{17}$ we can expect that the $\mathrm{N} 2 p$ orbitals of these nitrogen atoms participate in the strong chemical interaction with deposited aluminum atoms. This decreases the relative amplitude of this left $\mathrm{N} 1 s$ component and gives rise to the new reactive one R, appearing at lower $\mathrm{BE}$ as a consequence of strong charge transfer from $\mathrm{Al}$ to $\mathrm{N}$. Thus, we conclude that aluminum strong reacts with the aza nitrogen atoms and pyrrole carbon ones $\left(\mathrm{N}_{2}\right.$ and $\mathrm{C}_{1}$ correspondently).

This tendency probably indicates that on the first stage of $\mathrm{Al}$ deposition, strong chemical interaction of aluminum atoms with pyrrole nitrogen and pyrrole carbon atoms takes place in subsurface region, which modifies the CuPc molecules. As a result of this interaction, one have mixture of intact benzene rings, aluminum nitrides (AIN) and aluminum carbides $\left(\mathrm{Al}_{4} \mathrm{C}_{3}\right)$. By the way, $\mathrm{AlN}$ is a wide band gap $(6.2 \mathrm{eV})$ semiconductor material, has a hexagonal crystal structure, which is similar to the structure of the zinc sulfide, known as wurtzite. This is material for potential application in deep ultraviolet optoelectronics. As far to the aluminum carbide, it is known as a binary inorganic chemical compound that has a complex structure and is stable up to $1400^{\circ} \mathrm{C}$. Aluminum atoms of the compound are in a lattice of discrete carbon anions.

In addition to the fact, that the spectral shape of $\mathrm{B}$ component remains almost unchanged with aluminum coverage, this component of the $\mathrm{C} 1 s$ spectra show no shift with metal deposition. This can mean that in all range of Al deposition we observe no formation of noticeable interface dipole. However, we can suggest a different scenario. The formation of small aluminum clusters at the initial stage of the metal deposition on a thin film of the conjugated organic semiconductor may lead to positively charged $\mathrm{Al}$ clusters during photoemission spectroscopy investigations. This can cause the PES spectra shift to higher BE and thus compensate the shift induced by possible dipole formation.

\section{SUMMARY}

In summary, by TEM and by PES methods, we have investigated the morphology and electronic properties of hybrid systems consisting of $\mathrm{Al}$ nanoparticles blended into $\mathrm{CuPc}$ matrix for different nominal aluminum content. The experimental results demonstrate that Al NPs are formed by self-assembling in a well defined manner with a narrow diameter distribution which depends on the amount of aluminum that is evaporated onto the $\mathrm{CuPc}$ film. In general, $\mathrm{Al}$ nanoparticles form some particle distribution with size about 5-6 nm. With further deposition, one observes strong growth of individual grains. However, on the very first stage of metal deposition, one observed strong chemical reaction of $\mathrm{Al}$ atoms with atoms of $\mathrm{CuPc}$ in subsurface region. The results of our studies are summarized in Fig. 6. They clearly show that in a first stage upon aluminum deposition onto $\mathrm{CuPc}$, the aluminum atoms diffuse into the $\mathrm{CuPc}$ film and intensely react with the $\mathrm{CuPc}$ molecules. We find clear evidence of a charge transfer from $\mathrm{Al}$ to $\mathrm{CuPc}$ and we have been able to determine the lattice sites where $\mathrm{Al}$ ions sit. The finally at high coverage about $64 \AA$ the formation of metallic aluminum overlayer on $\mathrm{CuPc}$ thin film takes place.

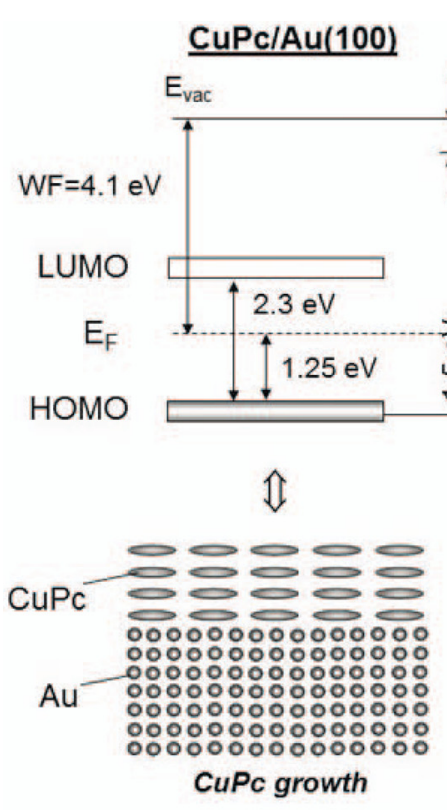

(a)

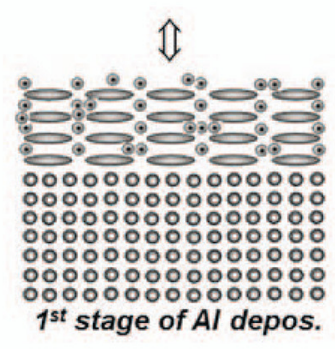

(b)

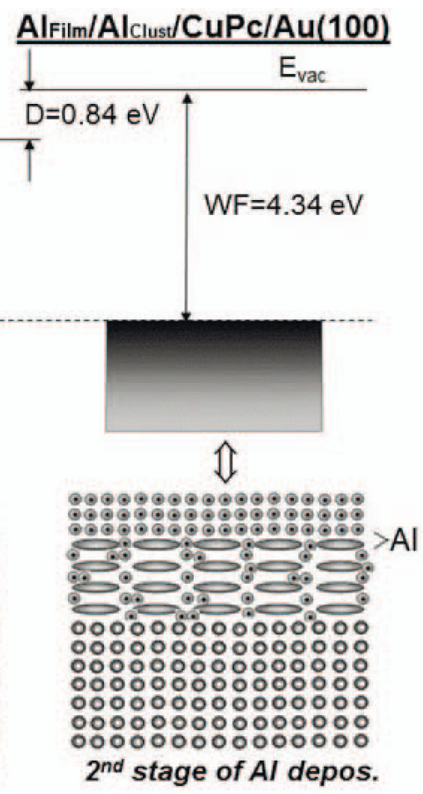

(c)

FIG. 6. Schematic representation of the energy levels derived from our experiment during aluminum deposition onto a CuPc film grown on Au(100). The situations in the different stages are evident from the diagram. 


\section{ACKNOWLEDGMENTS}

This work was supported by the RFBR Grant No. 1302-00818 and the BMBF-Project No. 05K12GU2, PSPElement No. U4606BMB1211.

${ }^{1}$ Z. Liu, A. A. Yasseri, J. S. Lindsey, and D. F. Bocian, Science 302, 1543 (2003).

${ }^{2}$ J. C. Scott, Science 304, 62 (2004).

${ }^{3}$ L. Bozano, B. Kean, M. Beinhoff, K. Carter, P. Rice, and J. Scott, Adv. Funct. Mater. 15, 1933 (2005).

${ }^{4}$ Y. Yang, J. Ouyang, L. Ma, R.-H. Tseng, and C.-W. Chu, Adv. Funct. Mater. 16, 1001 (2006).

${ }^{5}$ D. Tondelier, K. Lmimouni, D. Vuillaume, C. Fery, and G. Haas, Appl. Phys. Lett. 85, 5763 (2004).

${ }^{6}$ L. P. Ma, J. Liu, and Y. Yang, Appl. Phys. Lett. 80, 2997 (2002).

${ }^{7}$ L. P. Ma, J. Liu, S. Pyo, and Y. Yang, Appl. Phys. Lett. 80, 362 (2002).

${ }^{8}$ D. Prime, S. Paul, and P. W. Josephs-Franks, Philos. Trans. R. Soc., A 367, 4215 (2009).

${ }^{9}$ J. Ouyang, C.-W. Chu, C. R. Szmanda, L. Ma, and Y. Yang, Nature Mater. 3, 918 (2004).

${ }^{10}$ L. D. Bozano, B. W. Kean, V. R. Deline, J. R. Salem, and J. C. Scott, Appl. Phys. Lett. 84, 607 (2004).

${ }^{11}$ D. G. Fedak and N. A. Gjostein, Surf. Sci. 8, 77 (1967).

${ }^{12}$ M. V. Hove, R. Koestner, P. Stair, J. Bibérian, L. Kesmodel, I. Barto, and G. Somorjai, Surf. Sci. 103, 189 (1981).

${ }^{13}$ O. V. Molodtsova, M. Knupfer, Y. A. Ossipyan, and V. Y. Aristov, J. Appl. Phys. 104, 083704 (2008).

${ }^{14}$ T. Okajima, H. Fujimoto, M. Sumitomo, T. Araki, E. Ito, H. Ishii, Y. Ouchi, and K. Seki, Surf. Rev. Lett. 9, 441 (2002).

${ }^{15}$ H. Peisert, T. Schwieger, J. M. Auerhammer, M. Knupfer, M. S. Golden, J. Fink, P. R. Bressler, and M. Mast, J. Appl. Phys. 90, 466 (2001).

${ }^{16}$ G. Cabailh, J. Wells, I. McGovern, A. Vearey-Roberts, A. Bushell, and D. Evans, Appl. Surf. Sci. 234, 144 (2004).

${ }^{17}$ V. Y. Aristov, O. V. Molodtsova, C. Laubschat, V. M. Zhilin, I. M. Aristova, V. V. Kveder, and M. Knupfer, Appl. Phys. Lett. 97, 113103 (2010).
${ }^{18}$ A. C. Dürr, N. Koch, M. Kelsch, A. Rühm, J. Ghijsen, R. L. Johnson, J.-J. Pireaux, J. Schwartz, F. Schreiber, H. Dosch, and A. Kahn, Phys. Rev. B 68, 115428 (2003).

${ }^{19}$ N. Koch, A. C. Dürr, J. Ghijsen, R. L. Johnson, J. J. Pireaux, J. Schwartz, F. Schreiber, H. Dosch, and A. Kahn, Thin Solid Films 441, 145 (2003).

${ }^{20}$ V. Y. Aristov, O. V. Molodtsova, V. M. Zhilin, D. Vyalikh, and M. Knupfer, Phys. Rev. B 72, 165318 (2005).

${ }^{21}$ L. Ottaviano, S. D. Nardo, L. Lozzi, M. Passacantando, P. Picozzi, and S. Santucci, Surf. Sci. 373, 318 (1997).

${ }^{22}$ G. Dufour, C. Poncey, F. Rochet, H. Roulet, M. Sacchi, M. D. Santis, and M. D. Crescenzi, Surf. Sci. 319, 251 (1994).

${ }^{23}$ O. V. Molodtsova, V. M. Zhilin, D. V. Vyalikh, V. Y. Aristov, and M. Knupfer, J. Appl. Phys. 98, 093702 (2005).

${ }^{24}$ T. Schwieger, H. Peisert, M. S. Golden, M. Knupfer, and J. Fink, Phys. Rev. B 66, 155207 (2002).

${ }^{25}$ H. Peisert, M. Knupfer, and J. Fink, Surf. Sci. 515, 491 (2002).

${ }^{26}$ N. Papageorgiou, Y. Ferro, E. Salomon, A. Allouche, J. M. Layet, L. Giovanelli, and G. Le Lay, Phys. Rev. B 68, 235105 (2003).

${ }^{27}$ B. Brena, Y. Luo, M. Nyberg, S. Carniato, K. Nilson, Y. Alfredsson, J. Åhlund, N. Mårtensson, H. Siegbahn, and C. Puglia, Phys. Rev. B 70, 195214 (2004).

${ }^{28}$ Y. Hirose, A. Kahn, V. Aristov, P. Soukiassian, V. Bulovic, and S. R. Forrest, Phys. Rev. B 54, 13748 (1996).

${ }^{29}$ See http://www.kayelaby.npl.co.uk/atomic_and_nuclear_physics/ for information about the work functions of $\mathrm{Al}$ and $\mathrm{Au}$.

${ }^{30}$ D. K. Murti, L. J. Brillson, and J. H. Slowik, J. Vac. Sci. Technol. 20, 233 (1982).

${ }^{31}$ J. Ghijsen, L. H. Tjeng, J. van Elp, H. Eskes, J. Westerink, G. A. Sawatzky, and M. T. Czyzyk, Phys. Rev. B 38, 11322 (1988).

${ }^{32}$ S.-H. Su, C.-H. Chou, and M. Yokoyama, Electrochem. Solid-State Lett. 6, H17 (2003).

${ }^{33}$ V. Aristov, O. Molodtsova, Y. Ossipyan, B. Doyle, S. Nannarone, and M. Knupfer, Org. Electron. 10, 8 (2009).

${ }^{34}$ V. Y. Aristov, O. V. Molodtsova, Y. A. Ossipyan, B. P. Doyle, S. Nannarone, and M. Knupfer, Phys. Status Solidi A 206, 2763 (2009).

${ }^{35}$ C. Shen, A. Kahn, and J. Schwartz, J. Appl. Phys. 90, 6236 (2001).

${ }^{36}$ M.-S. Liao and S. Scheiner, J. Chem. Phys. 114, 9780 (2001). 